\title{
Getting hotter by heating less: How driven granular materials dissipate energy in excess
}

\author{
A. Plati $\odot,{ }^{1,2}$ L. de Arcangelis $\odot,{ }^{3}$ A. Gnoli $\odot,{ }^{1,2}$ E. Lippiello $\odot,{ }^{4, *}$ A. Puglisi $\odot,{ }^{1,2,5}$ and A. Sarracino $\circledast^{3}$ \\ ${ }^{1}$ Department of Physics, University of Rome Sapienza, P. le Aldo Moro 2, 00185 Rome, Italy \\ ${ }^{2}$ Institute for Complex Systems-CNR, P. le Aldo Moro 2, 00185 Rome, Italy \\ ${ }^{3}$ Department of Engineering, University of Campania "Luigi Vanvitelli," 81031 Aversa (Caserta), Italy \\ ${ }^{4}$ Department of Mathematics and Physics, University of Campania "Luigi Vanvitelli," 81100 Caserta, Italy \\ ${ }^{5}$ INFN, University of Rome Tor Vergata, Via della Ricerca Scientifica 1, 00133 Rome, Italy
}

(Received 5 April 2020; revised 15 July 2020; accepted 17 December 2020; published 6 January 2021; corrected 27 January 2021)

\begin{abstract}
We investigate how the kinetic energy acquired by a dense granular system driven by an external vibration depends on the input energy. Our focus is on the dependence of the granular behavior on two main parameters: frequency and vibration amplitude. We find that there exists an optimal forcing frequency at which the system reaches the maximal kinetic energy: if the input energy is increased beyond this threshold, the system dissipates more and more energy and recovers a colder and more viscous state. Quite surprisingly, the nonmonotonic behavior is found for vibration amplitudes which are sufficiently low to keep the system always in contact with the driving oscillating plate. Studying dissipative properties of the system, we unveil a striking difference between this nonmonotonic behavior and a standard resonance mechanism. This feature is also observed at the microscopic scale of the single-grain dynamics and can be interpreted as an instance of negative specific heat. An analytically solvable model based on a generalized forced-damped oscillator well reproduces the observed phenomenology, illustrating the role of the competing effects of forcing and dissipation.
\end{abstract}

DOI: 10.1103/PhysRevResearch.3.013011

\section{INTRODUCTION}

The fascination of granular systems relies on their rich phenomenology, which eludes standard statistical physics [1]. Examples of surprising behaviors were reported in experiments and numerical simulations: the Brazil-nut effect [2], the ratchet effect $[3,4]$, spontaneous segregation of mixtures [5], nonequilibrium phase transitions [6], anomalous thermal convection [7,8], and the Kovacs-like memory effect [9] (see also [10]). These features are ascribed to the dissipative nature of these systems, which generally cannot be treated via the introduction of effective parameters [11]. A fundamental open question is how the dissipation mechanisms relevant for different behaviors are related to the external energy injection. These mechanisms involve several scales, from particle-particle collisions to the interaction with boundaries.

The relation between the system kinetic energy $K$ and the input energy $S$ involves the nonequilibrium response beyond the linear regime. In general, nonmonotonic behaviors have been observed in different models, from driven tracers in crowding environments (negative differential mobility) [12-17], to systems showing a negative specific heat due to long-range interactions [18-21], to the presence of

\footnotetext{
*eugenio.lippiello@unicampania.it
}

Published by the American Physical Society under the terms of the Creative Commons Attribution 4.0 International license. Further distribution of this work must maintain attribution to the author(s) and the published article's title, journal citation, and DOI. baths at different temperatures [22,23]. Similar effects have also been observed in force-free cooling granular gases of aggregating particles [24], where the granular temperature can increase while the system energy decreases due to dissipative collisions.

These behaviors affect fluidization properties of the medium, with important consequences in industrial applications [25], where usually energy is fed via mechanical vibrations with frequency $f$ and amplitude $A$. In some cases, the relation $K \sim S^{\alpha}$, with $S \sim(A f)^{2}$, has been derived [26-29]. Experimental studies have focused on the specific role of the forcing mechanisms, such as the vibration amplitude, frequency, and velocity [30-47]. In particular, an optimal frequency for energy transfer is found when the system is in a bouncing-bed state and $A$ and $f$ are varied, keeping $S$ fixed [30,31]. In this state the granular medium detaches from the driving plate and a resonant behavior is achieved via a synchronization between plate vibration and bed bouncing.

In this paper we consider vibration amplitudes low enough to keep the granular system always in contact with the oscillating plate. Our main result is that, even in this regime, if the input energy fed into the system is increased by increasing $f$ (keeping $A$ fixed), nonmonotonic behavior is observed in both the kinetic energy of a driven vane immersed in the medium and in the kinetic energy of the granular medium itself. We show that there is an optimal frequency where the system reaches a maximum kinetic energy. Differently from previous results $[30,31]$, our system is not in a bouncing-bed or resonant state, and the granular internal energy is nonmonotonic with $f$, even if the energy input always increases. Our results can be interpreted as an example of negative specific 


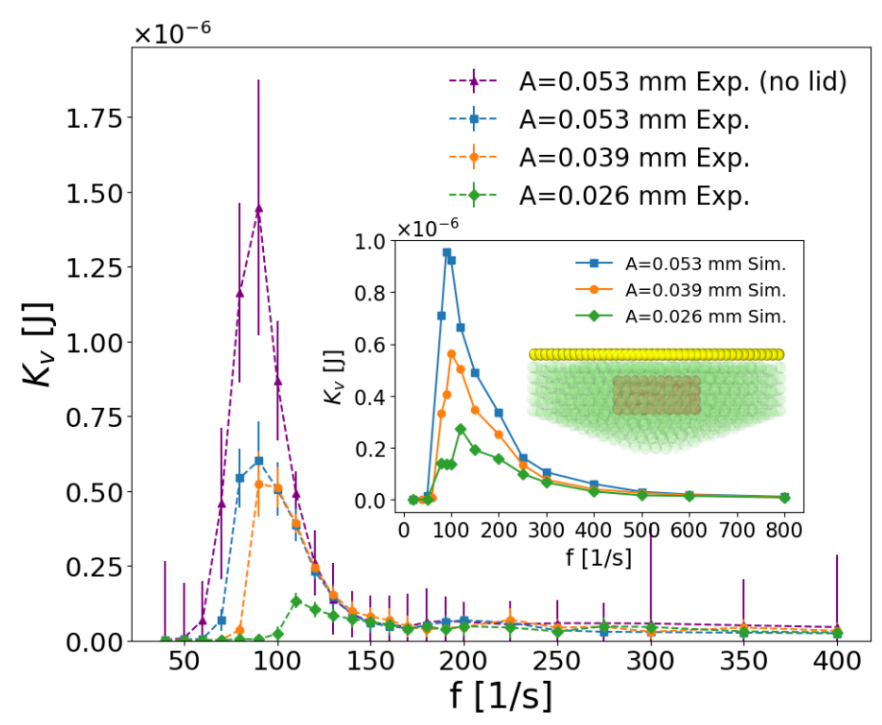

FIG. 1. Vane kinetic energy $K_{v}$ in experiments as a function of $f$, for different values of $A$. Error bars are \pm 1 standard deviation. Inset: $K_{v}$ measured in numerical simulations. Sketch of the system setup: green circles show granular particles, red circles represent the vane, and yellow circles the lid. Numerical parameters are $k_{n}=12 \times$ $10^{7} \mathrm{~Pa}$ and $\gamma_{n}=2.9 \times 10^{7}(\mathrm{~ms})^{-1}$.

heat, shedding light on the findings reported in [24]: Here, we investigate the complementary process, where the granular temperature decreases when the input energy increases.

The paper is structured as follows. In Sec. II we describe the experimental setup and the numerical model. In Sec. III we discuss the nonmonotonic behavior of the system energy as a function of the input energy, which represents the main result of the paper. Section IV is devoted to the study of the vane angular velocity, which shows a similar nonmonotonic behavior, related to the viscous properties of the granular medium. In Sec. V we present an analysis of the single-particle dynamics, which provides further details on the physical mechanisms underlying the observed phenomenology. The proposed theoretical model of a generalized driven-damped oscillator is described in Sec. VI. In Sec. VII we compare our findings with previous results, pointing out the main differences. Finally, in Sec. VIII we draw some conclusions on the effects and mechanisms observed in our system. The Appendix provides details on the numerical model.

\section{EXPERIMENTAL SETUP AND NUMERICAL MODEL}

Our experimental setup is illustrated in Fig. 1 (see also Ref. [33]). The granular medium is made up of $N=2600$ steel spheres (diameter $d=4 \mathrm{~mm}$, mass $m=0.27 \mathrm{~g}$ ) contained in a conical-shaped floor cylinder (diameter, $90 \mathrm{~mm}$; minimum height, $28.5 \mathrm{~mm}$; maximum height, $47.5 \mathrm{~mm}$ ), which enhances the energy transfer from vertical to horizontal directions. A rigid lid (mass $M_{\text {top }}=218 \mathrm{~g}$ ) covers the system, to confine particles and to allow for a spectral analysis of the system oscillations. A vertical vibration is imposed on the coordinate of the container bottom, $z_{p}(t)$ :

$$
z_{p}(t)=A \sin (2 \pi f t)
$$

A Plexiglas vane (height, $15 \mathrm{~mm}$; width, $6 \mathrm{~mm}$; length, $35 \mathrm{~mm}$ ) is suspended in the system. The vane can only rotate around the vertical axis, subjected to a constant torque $\mathcal{T}=6 \times 10^{-3} \mathrm{~N} \mathrm{~m}$.

The system is simulated by the LAMMPS package [48]. The interaction among grains is described via the nonlinear Hertz-Mindlin (HM) model [49,50]; see the Appendix for details. The relevant parameters are the stiffness of the nonlinear contact $k_{n}$ and the viscous damping coefficient $\gamma_{n}$, accounting for dissipative interactions. The geometry of the system and numerical parameters are chosen to reproduce our experimental setup: The lid is made up of 1773 granular particles glued together and the rotating vane is made up of $4 \times 10$ particles glued together and overlapping by half a radius.

\section{OPTIMAL FORCING FREQUENCY AND ROLE OF DISSIPATION}

In the main panel in Fig. 1 we report the vane kinetic energy contribution due to fluctuations, $K_{v}=I\left[\left\langle\Omega^{2}\right\rangle-\right.$ $\left.\langle\Omega\rangle^{2}\right] / 2$ [51], where $I=353 \mathrm{~g} \mathrm{~mm}^{2}$ is the momentum of inertia and $\Omega$ the angular velocity, measured in experiments as a function of $f$ for different values of $A$. We also show a case with no lid to demonstrate the robustness of the observed behavior. In the inset we report results of numerical simulations showing that the model well reproduces the nonmonotonic behavior of the real system. In the following, we are mainly interested in the total kinetic energy of the granular medium and in the dynamics of single grains, and therefore we remove the vane in some simulations of the numerical model [52]. Numerical simulations allow us to investigate the granular kinetic energy and single-grain motion, exploring a wider range of vibration frequencies, $f \in(0,1000] \mathrm{Hz}$, with respect to the experiments.

As shown in Fig. 1, the vane kinetic energy is a nonmonotonic function of $f$ (at fixed $A$ ): $K_{v}$ increases abruptly from 0 to a finite value at a frequency threshold $f_{1}$, related to the detachment condition [39], $2 \pi f_{1}=\sqrt{g / A}$. Then, after a maximum, $K_{v}$ starts to decrease, signaling that the granular medium leaves the state of maximal fluidization, hindering the motion of the vane by an increased effective viscosity. One can define a so-called friction recovery frequency, $f_{2}>f_{1}$, at which the system kinetic energy decays to about $1 / 2$ of its maximum value. This behavior is related to dissipation mechanisms of the granular medium that depend on the viscoelastic properties of the material.

To better characterize the intrinsic behavior of the granular medium and its response to the external forcing, we focus on the total translational kinetic energy (the rotational kinetic energy being negligible; see below) $K=\sum_{i=1}^{N} m\left\langle\boldsymbol{v}_{i}^{2}\right\rangle / 2$, where $\boldsymbol{v}_{i}$ is the grain velocity, in the absence of the suspended vane. Therefore, we introduce the adimensional quantity describing the energy input, $S=(2 \pi f)^{2} A^{2} /(g d)$.

In Fig. 2 we report $K(S)$, varying $f$ at fixed $A$, for several values of $\gamma_{n}$. The nonmonotonic behavior shows that the granular system reaches the maximal kinetic temperature at an optimal value of the input energy. When the input energy exceeds a certain threshold, related to $f_{2}$, the system cools down because the dissipation effects increase. A key result is represented by the maximum position of the kinetic energy 


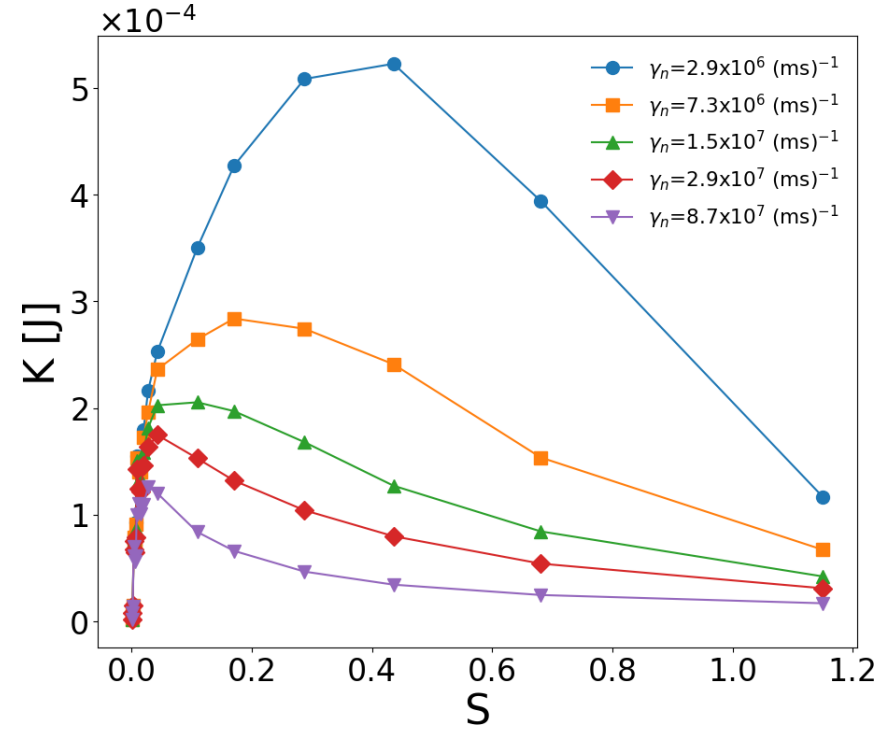

FIG. 2. Total kinetic energy $K$ of the granular medium versus $S$, varying $f$ at fixed $A$, for several values of $\gamma_{n}$, with $k_{n}=6.1 \times 10^{7} \mathrm{~Pa}$ and $A=0.026 \mathrm{~mm}$.

for different values of the viscous damping coefficient $\gamma_{n}$. We find that the peak shifts to the left when the dissipation in the system is increased. It is interesting to note that this shift does not occur if we vary $k_{n}$, signaling that the friction-recovery mechanism is not governed by the elastic contribution of the interaction [see Fig. 3(b)]. Therefore the system can transfer more energy to the grain motion when it is vibrated at an optimal frequency. If the frequency increases, the overall external energy injected is higher but the dissipation mechanisms become dominant and the granular kinetic temperature decreases. This behavior can be interpreted as an instance of negative specific heat [22,24], occurring due to the subtle interplay between forcing and dissipation.

Conversely, if we increase $S$ by increasing $A$ at fixed $f$, we find a monotonic behavior. Namely, dissipative mechanisms are not activated and the kinetic energy keeps increasing with the input energy as shown in Fig. 4. These features are a consequence of the permanent contact with the driving plate, which makes dissipation dominant at high frequencies. This is a novel phenomenon different from the resonant behavior in the bouncing-bed state clearly described in Refs. [30,31], where the optimal frequency is an increasing function of the dissipation. Striking differences are also provided by the spectral analysis of the top-plate oscillations, which indicates that, in our system, the energy transfer is not maximized in the most coherent states (see Sec. VII C).

\section{VANE AVERAGE VELOCITY}

Another crucial quantity in our experimental/numerical setup is the average angular velocity $\langle\Omega\rangle$ of the vane in the steady state. While the variance is related to the kinetic energy of the system, the mean value of $\Omega$ is proportional to the inverse of the viscosity acting on the tracer during its motion. In the following we present the numerical study of $\langle\Omega\rangle$ and $K$ with particular attention paid to the role played by the
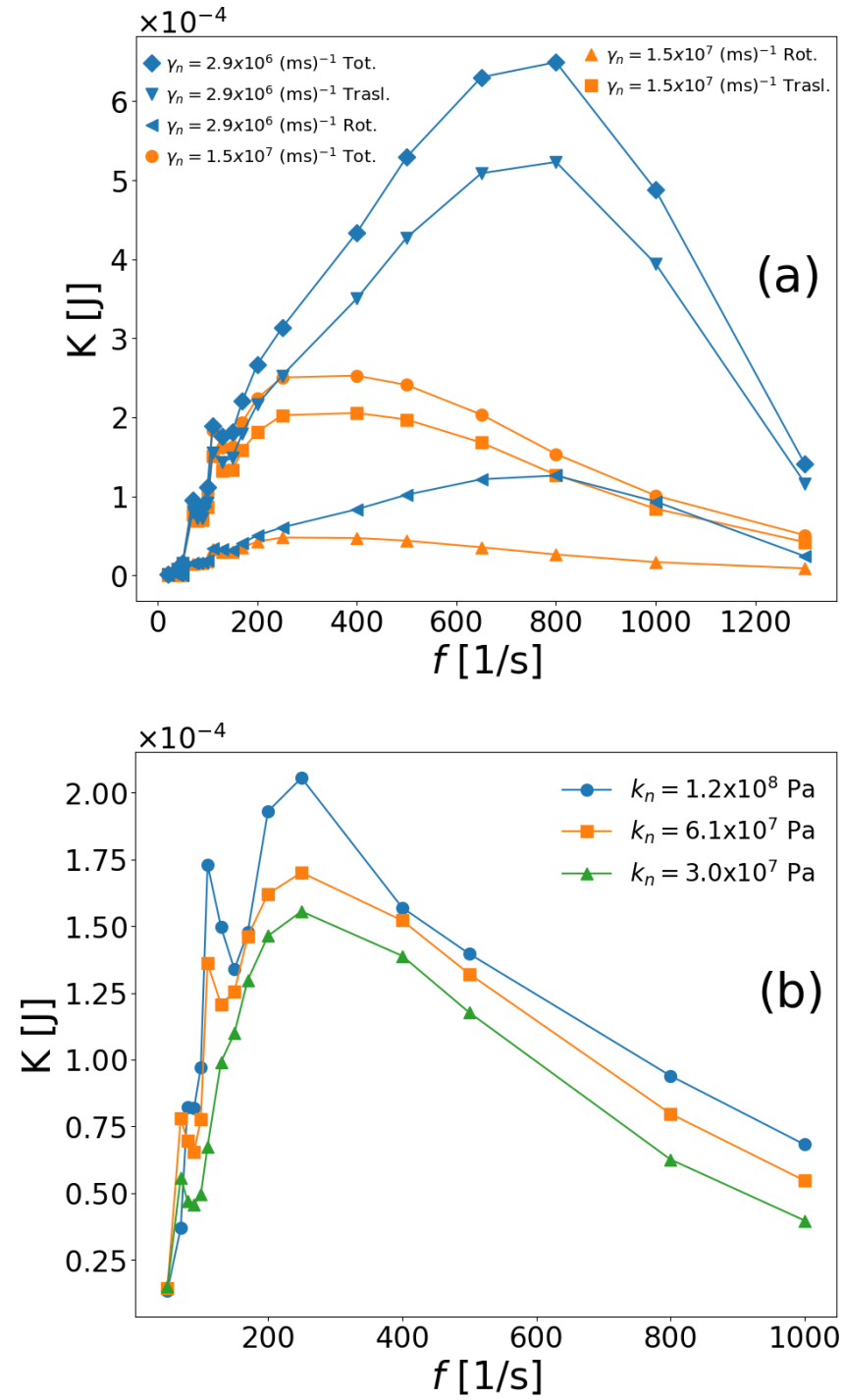

FIG. 3. Mean translational kinetic energy of the whole system $K$ versus the driving frequency $f$ at fixed $A=0.026 \mathrm{~mm}$ (simulations without the vane). (a) Comparison between the translational kinetic energy, the rotational energy, and the total energy (translational plus rotational). We show two curves reported in Fig. 2 as a function of $f$, instead of $S$. The rotational degrees of freedom follow the same nonmonotonic behavior but with smaller absolute values, so that their contribution does not affect the qualitative behavior of the total kinetic energy. (b) Study of the mean kinetic energy for different values of nonlinear stiffness: $k_{n}$ is varied at fixed $\gamma_{n}=2.9 \times 10^{7}$ $(\mathrm{ms})^{-1}$. The three curves have the same shape (the peak position does not change) but are vertically ordered by $k_{n}$. This implies a higher kinetic energy and therefore less dissipation in the system with a higher stiffness.

parameters $k_{n}$ and $\gamma_{n}$ of the HM model in the characterization of its behavior. For an experimental study of this observable in the same setup see [33].

In Fig. 4(b) we show $\langle\Omega\rangle$ as a function of $S$ by increasing $f$ at fixed $A$, and vice versa. We find a behavior very similar to that of $K$ reported in the previous section; the only qualitative difference is that $\langle\Omega\rangle$ versus $A$ reaches a constant value, while $K$ versus $A$ keeps increasing also at higher amplitudes 

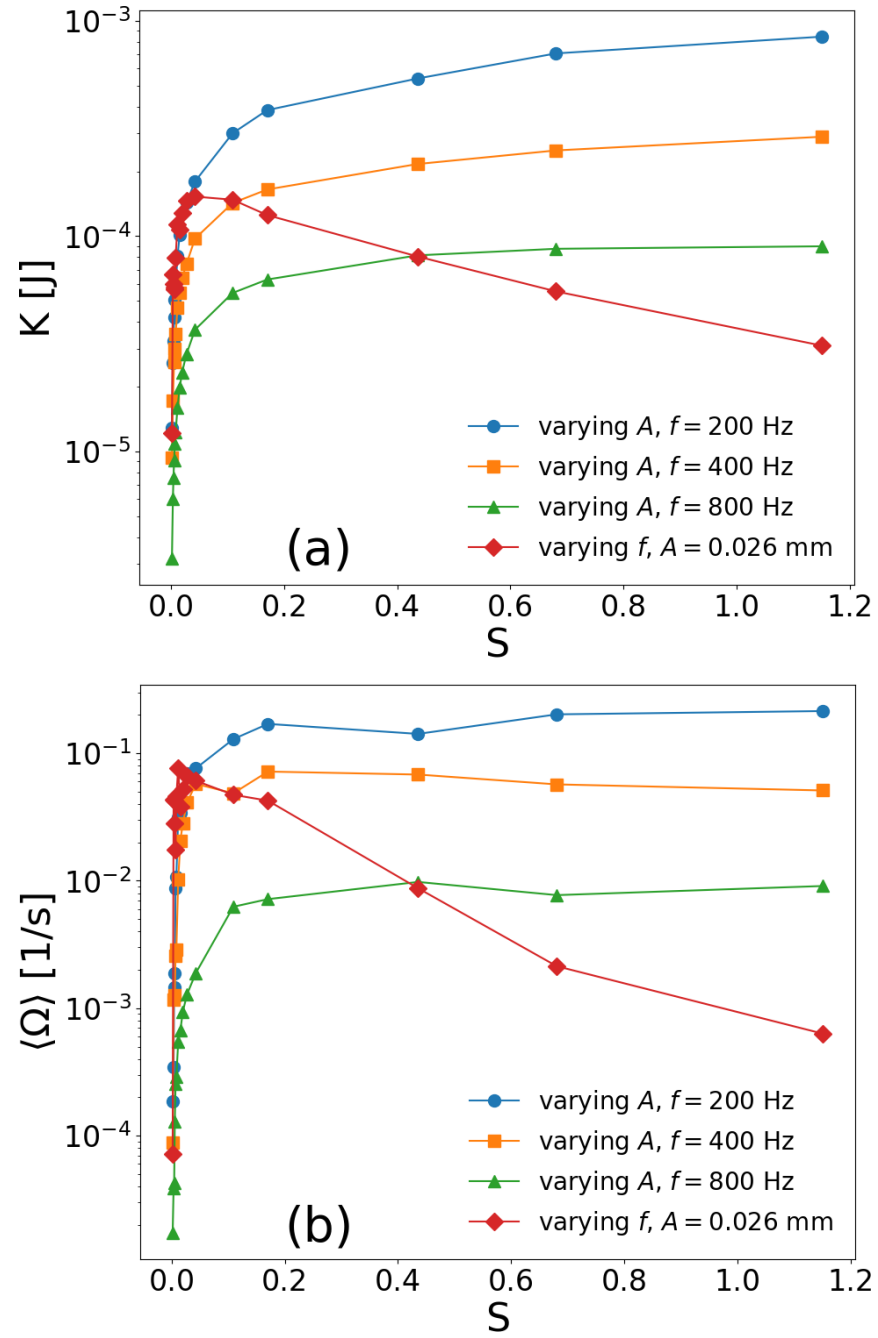

FIG. 4. Total kinetic energy $K$ of the grains (a) and mean angular velocity $\langle\Omega\rangle$ of the blade (b) versus $S$, varying $A$ for three fixed frequencies $(200,400$, and $800 \mathrm{~Hz})$ and varying $f$ for one fixed amplitude $(A=0.026 \mathrm{~mm})$. Parameters are $k_{n}=6.1 \times 10^{7} \mathrm{~Pa}$ and $\gamma_{n}=2.9 \times 10^{7}(\mathrm{~ms})^{-1}$.

[compare with Fig. 4(a)]. To better understand the role of the interaction parameters we concentrate on $\langle\Omega\rangle(f)$ at a constant amplitude. In Figs. 5(a) and 5(b) we vary $\gamma_{n}$ at fixed $k_{n}$, while in Fig. 5(c) we vary $k_{n}$ at fixed $\gamma_{n}$. We see that the parameter $k_{n}$ changes the typical shape of the curves near $f_{1}$ : we have a sudden peak when $k_{n}$ is high and a smoother behavior when it is low. In Fig. 5(b) we observe that at a lower $k_{n}$, reducing $\gamma_{n}$ clearly slows down the friction recovery. On the other hand, at a higher $k_{n}$ [Fig. 5(a)] we see the same effect but without a significant change in the height of the peak. This can be interpreted intuitively observing that a higher stiffness reduces the effect of the viscous damping during the collision. In Fig. 5(c) we see that also an increase in $k_{n}$ implies an increase in the recovery frequency, but this effect is less pronounced with respect to the one obtained with decreasing $\gamma_{n}$. We can conclude that $f_{2}$ is mostly ruled by the dissipation through $\gamma_{n}$, in agreement with previous experimental results [33].

To sum up, a comparison between Figs. 5(a), 5(b), and 3(a) shows that the behavior of both $\langle\Omega\rangle$ (a rheological response)
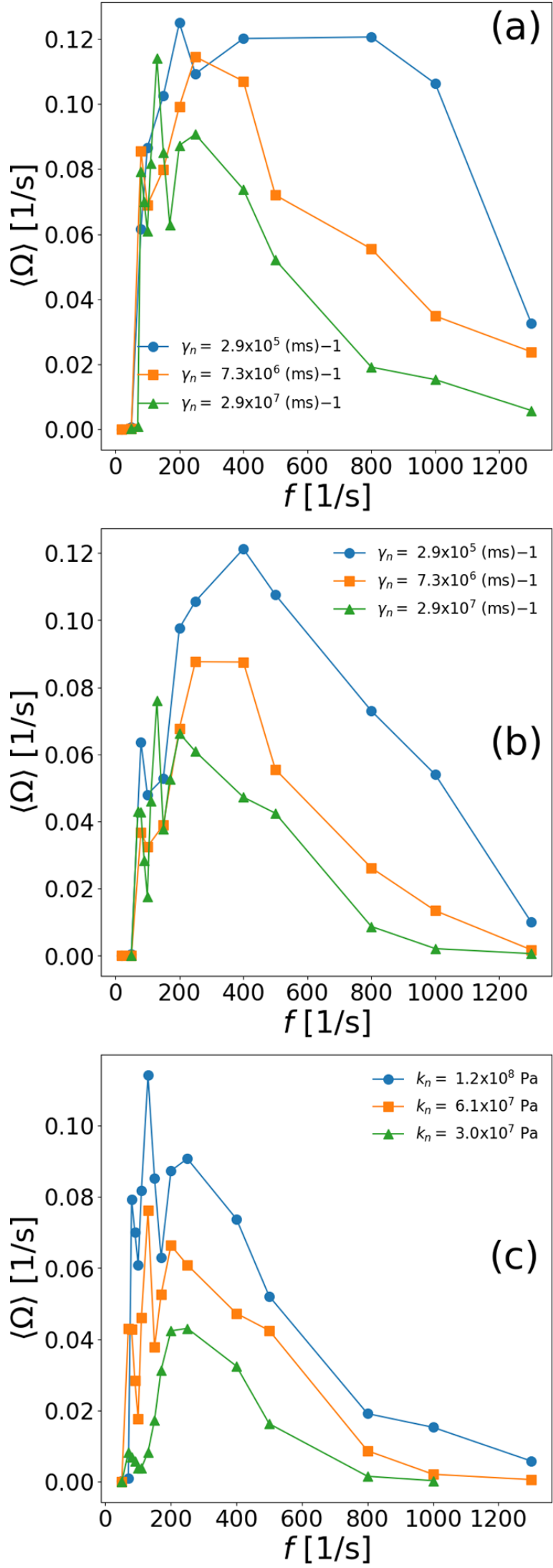

FIG. 5. Mean angular velocity of the blade $\langle\Omega\rangle$ versus the driving frequency $f$. (a) $\gamma_{n}$ is varied with a fixed $k_{n}=1.5 \times 10^{8} \mathrm{~Pa}$. (b) $\gamma_{n}$ with a fixed $k_{n}=6.1 \times 10^{7} \mathrm{~Pa}$. (c) $k_{n}$ is varied with a fixed $\gamma_{n}=$ $2.9 \times 10^{7}(\mathrm{~ms})^{-1}$. 


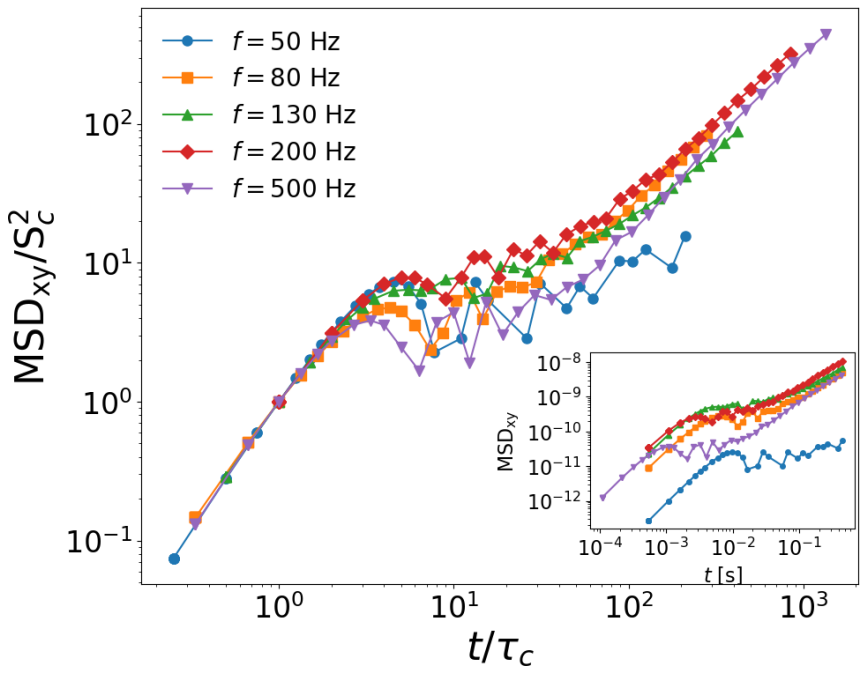

FIG. 6. Rescaled single-particle MSD on the $X Y$ plane versus the rescaled time, for different values of the driving frequency. Inset: Single-particle MSD on the $X Y$ plane versus the time. Simulation parameters are $A=0.026 \mathrm{~mm}, k_{n}=6.1 \times 10^{7} \mathrm{~Pa}$, and $\gamma_{n}=2.9 \times$ $10^{7}(\mathrm{~ms})^{-1}$.

and $K$ (a thermodynamic observable) as a function of $f$ is similarly affected by the change in $\gamma_{n}$. On the contrary, from Figs. 5(c) and 3(b), it is clear that changing $k_{n}$ affects $\langle\Omega\rangle$ more than $K$.

\section{ANALYSIS OF THE SINGLE-PARTICLE DYNAMICS}

The macroscopic features described above can be related to the microscopic properties by investigating the single-grain dynamics. Typically, in dense systems, the diffusive motion of a single particle is hindered by the presence of many surrounding particles, inducing a cage effect [53-55]. Focusing on the mean squared displacement (MSD) of a particle, one observes that, after a ballistic motion at short times, a plateau develops, signaling that the particle is trapped inside a cage. Then, at longer times, the particle manages to explore a larger region of the system and its MSD increases linearly in time. The relevant quantities are the cage size $S_{c}$ and the trapping time inside a cage $\tau_{c}$, both estimated from the first point of the MSD after the ballistic regime. This picture is fully supported by Fig. 6, where we show the MSD (averaged over about 20 particles randomly chosen in the system) on the horizontal $X Y$ plane, the plane relevant for the vane's motion. Rescaling time by the trapping time $\tau_{c}$ and the MSD by the squared cage size, we find that the curves collapse.

The nonmonotonic behaviors described above for $K_{v}$ and $K$ can be rationalized by the study of $S_{c}$ and $\tau_{c}$, as reported in the insets in Fig. 7. We find that $\tau_{c}$ is a decreasing function of the frequency, $\tau_{c} \sim f^{-0.67}$, whereas $S_{c}$ is a nonmonotonic function of $S$, varying $f$ at fixed $A$, with a maximum at $S \simeq 10^{-2}$. At low frequencies the cage size tends to be very small but the particles require a long time to be trapped. Conversely, at high frequencies the cages are still small but the particles are trapped in a short time. Moreover, at low frequencies, since the system is weakly perturbed by the input energy, a particle is able to explore larger regions for increasing $S$. However, for larger $S$ (increased through $f$ ), collisions become more

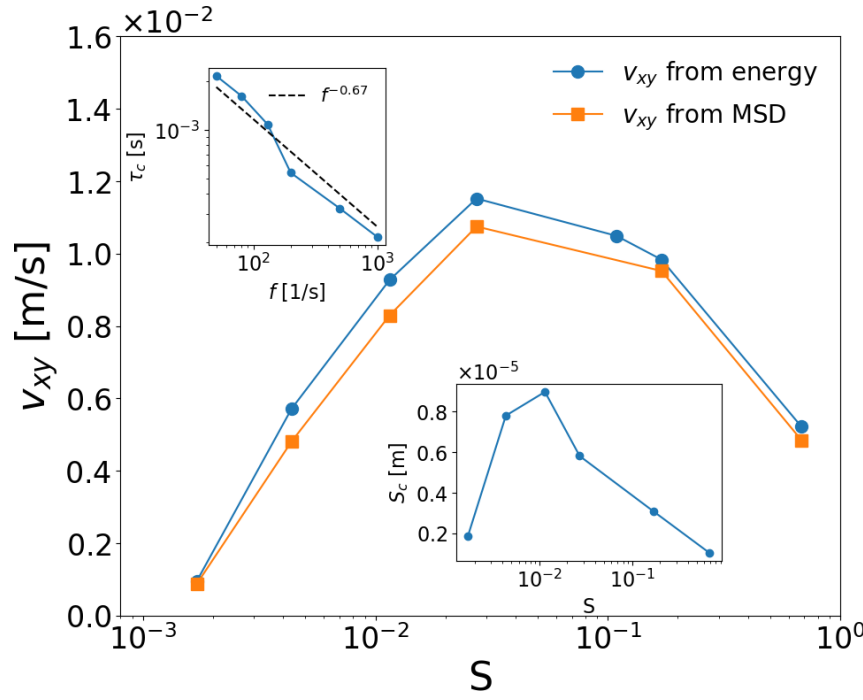

FIG. 7. Comparison between the average particle speed on the $X Y$ plane obtained from the mean kinetic energy of the whole system and from the ratio between the cage size $S_{c}$ and the trapping time $\tau_{c}$. Bottom inset: $S_{c}$ as a function of the input energy $S$, varying $f$ at fixed $A$. Top inset: $\tau_{c}$ as a function of $f$. Same parameters as in Fig. 6 .

frequent and the explored cage region decreases accordingly. If we estimate the average particle speed $v_{X Y}$ on the $X Y$ plane as $v_{X Y} \sim S_{c} / \tau_{c}$, we find a nonmonotonic behavior, in agreement with the behavior of $K$ (Fig. 7). This analysis shows that the single-particle dynamics reflects the same phenomenology occurring at the macroscopic scale.

Moreover, the nonmonotonic behavior of $S_{c}$ could be related to a change in the effective number of degrees of freedom in the system as the input energy is varied, in agreement with the explanation of negative specific heat suggested in Ref. [24] for cooling granular gases of aggregating particles. In that case, although the total energy of the system decreases due to dissipative collisions, the total number of clusters can decrease more rapidly due to agglomeration, producing an increase in the energy per particle.

We show in Fig. 8 the rescaled MSD on the $X Y$ plane averaged over 20 particles and the relative cage size $S_{c}$ for different values of $A$. Here we see that the collapse is very good up to time $t / \tau_{c} \sim 1$ (so the trapping time $\tau_{c}$ does not vary with $A$ ), while $S_{c}$ (and consequently the speed $v_{X Y}=S_{c} / \tau_{c}$ ) increases monotonically with the driving amplitude. The latter results show that the same phenomenology as a function of $A$ at a fixed frequency is present both at the macroscopic scale $(\langle\Omega\rangle$ and $K)$ and at the single-particle one $\left(v_{X Y}\right)$.

\section{THE GENERALIZED DRIVEN-DAMPED OSCILLATOR: A SINGLE-PARTICLE PERSPECTIVE}

Here we present a generalized model of a driven-damped oscillator that reproduces qualitatively the phenomenology of $K$ studied in the simulations. In particular, our model shows (i) a nonmonotonic behavior of the energy as a function of the driving frequency $f$ with a maximum in $f^{*}$, (ii) an increasing behavior of the energy as a function of the driving amplitude $A$, and (iii) a shift to the left of the frequency $f^{*}$ when the dissipation in the system is increased. 

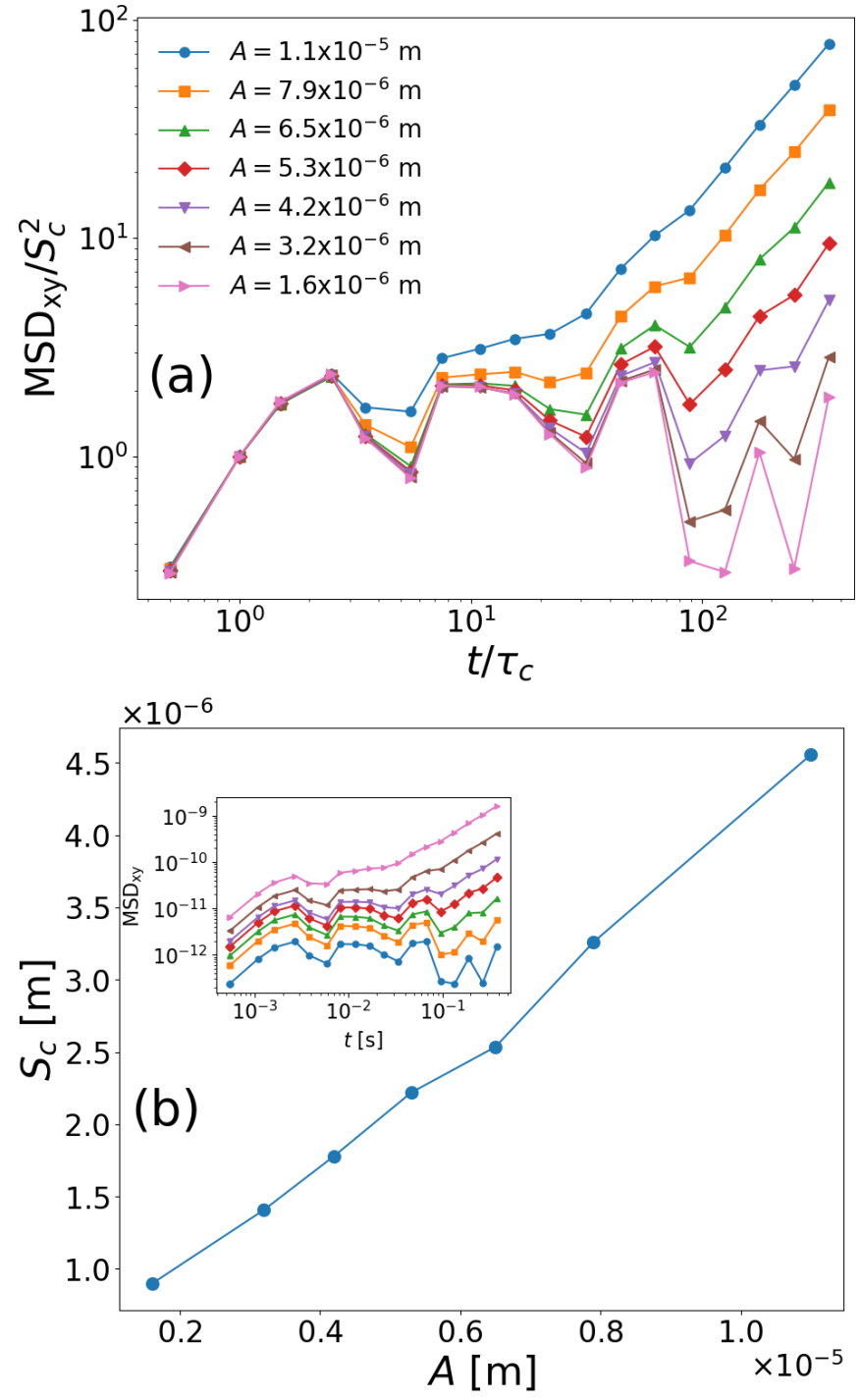

FIG. 8. (a) Single-particle MSD on the $X Y$ plane rescaled by $S_{c}^{2}$ as a function of the rescaled time $t / \tau_{c}$. Different curves correspond to different driving amplitudes, while the frequency is fixed at $f=200 \mathrm{~Hz}$. (b) Size of the cage $S_{c}$ versus the driving amplitude $A$. Inset: MSD not rescaled. Simulations with $k_{n}=6.1 \times 10^{7} \mathrm{~Pa}$ and $\gamma_{n}=2.9 \times 10^{7}(\mathrm{~ms})^{-1}$.

The model is obtained starting from an equation of motion for a generic particle in the system and then assuming that the energy of the whole system follows the same behavior as that of the single particle. This assumption is consistent with the previous analysis that shows a common phenomenology of single-particle quantities and macroscopic ones. In addition, the same assumption is done in positron emission particle tracking (PEPT) experiments, where the time-averaged behavior of a single tracked particle is considered representative of that of the global system [30].

\section{A. Newton equation for a caged single particle}

The granular system is very dense in all regimes of shaking, so the short-time dynamics of the grains is expected to take place inside their cages formed by the surrounding

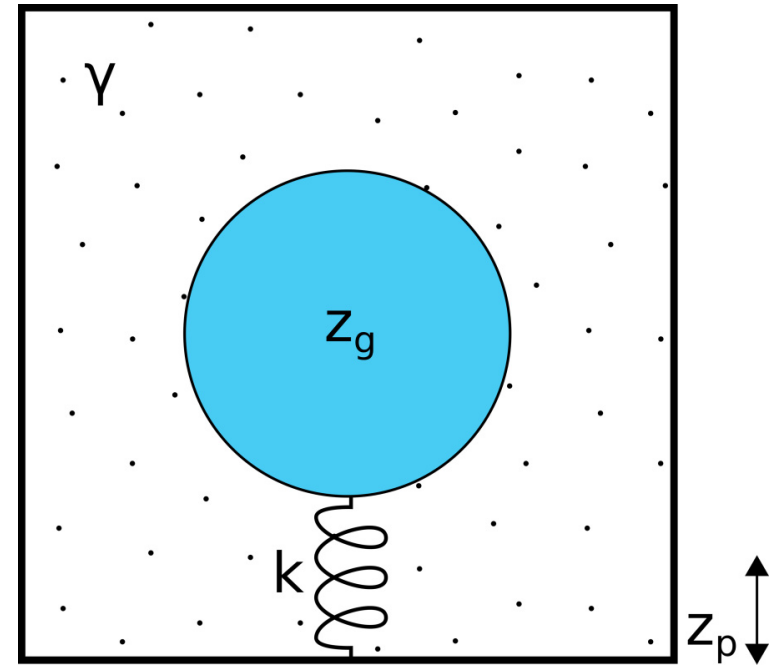

FIG. 9. Coarse-grained description of the single-particle dynamics. The springs $k$ and the viscosity $\gamma$ represent the cage that mediates the vibration of the shaker.

particles. This short-time dynamics presents multiple relevant time scales such as the one represented by the integration time $d t\left(\tau_{1} \sim 10^{-5} \mathrm{~s}\right)$, which is a fraction of the duration of a single collision, and the one fixed by the inverse of the driving frequency, $\tau_{d}=1 / f \sim 10^{-3}-10^{-1} \mathrm{~s}$. A third one is represented by the mean time between two collisions, reported in Fig. 7 (but also observed for the $X Y Z$ diffusion), which spans values in the range $\tau_{c} \sim 10^{-4}-10^{-3} \mathrm{~s}$. These time scales are thus ordered as $\tau_{1}<\tau_{c}<\tau_{d}$ for all the cases presented in our study.

In order to estimate the mean kinetic energy over many collisions, we are interested in a time coarse-graining on a scale larger than $\tau_{c}$. At the same time, we want to write a differential equation for the coordinates of a particle in which the shaker dynamics appears as an external driving. In view of these arguments, we concentrate on time scales larger than $\tau_{c}$ but smaller than $\tau_{d}$. To make our model as simple as possible we consider only the motion in the $z$ direction. Therefore we consider a single particle of mass $m$ confined in a onedimensional cage that consists of one spring of stiffness $k$ with a resting length $l_{0}$ connected with the bottom of a vibrating box. The latter really represents the experimental/numerical container that oscillates following $z_{p}(t)=A \cos (2 \pi f t)$. The fact that the cage is actually made of fast-vibrating particles also induces an effective viscosity with coefficient $\gamma$.

Our simplified coarse-grained description is sketched in Fig. 9, where we refer to the coordinate of the grain as $z_{g}$. We recall here that the coefficients of the HM model used in the simulation $\left(k_{n}\right.$ and $\left.\gamma_{n}\right)$ depend on the material properties and act on the fast time scale $\tau_{1}$. The way in which they are connected with the effective viscosity $\gamma$ and stiffness $k$ is not trivial. A reasonable value for $k$ can be estimated considering that the modeled spring is actually made up of a column of grains. Every grain with radius $r$ and Young modulus $Y$ can be thought of as a microscopic vertical spring with an elastic constant $\tilde{k}$ given by $\tilde{k}=\pi Y r / 2$ (for simplicity we consider the grains as cylinders instead of spheres). Now the effective stiffness of the column results from the parallel of a mean 
number $\bar{n}$ of such microscopic springs: $k=\tilde{k} / \bar{n}$. For the parameters of our simulations and fixing $\bar{n}=4.5$ we find that $k \sim O\left(10^{5}\right) \mathrm{N} / \mathrm{m}$. Regarding the effective viscosity, we use an ansatz in the following. Looking at Fig. 9, it is straightforward to write for $z_{g}$

$$
\ddot{z}_{g}(t)+4 \pi f_{s} \dot{z}_{g}(t)+\left(2 \pi f_{k}\right)^{2} \xi(t)=0,
$$

where $\xi(t)=z_{g}-z_{p}-l_{0}, \quad 2 \pi f_{s}=\gamma /(2 m)$, and $2 \pi f_{k}=$ $\sqrt{k / m} \sim O\left(10^{4}\right) \mathrm{s}^{-1}$.

Now we come to the crucial hypothesis of our approach, i.e., the ansatz on $f_{s}$. Some previous studies $[30,33]$ suggest that the dissipation of energy due to interparticle collisions increases significantly when the driving frequency $f$ increases. This is also visible in our data in Fig. 7, where we see that $\tau_{c}$ becomes smaller for increasing $f$. Indeed, a reduction of the time between collisions means an increase in the number of dissipative events in the system (i.e., the collisions themselves). The simplest way to insert this dependence of the internal dissipation on the external driving is to take $f_{s}$ as an increasing function of $f: f_{s}=a f^{\alpha}$ with $a, \alpha>0$. Bringing the variable $z_{p}(t)$ contained in $\xi(t)$ to the right-hand side of Eq. (2) and adding gravity, we obtain the following equation:

$$
\begin{aligned}
& \ddot{z}_{g}(t)+4 \pi a f^{\alpha} \dot{z}_{g}(t)+\left(2 \pi f_{k}\right)^{2} z_{g}(t) \\
& \quad=\left(2 \pi f_{k}\right)^{2} A \cos (2 \pi f t)+\left(2 \pi f_{k}\right)^{2} l_{0}-g .
\end{aligned}
$$

This is the equation for a driven-damped harmonic oscillator with characteristic frequency $f_{k}$, viscous constant $4 \pi a f^{\alpha}$, and external driving $\left(2 \pi f_{k}\right)^{2} A \cos (2 \pi f t)$ that oscillates around the equilibrium position $z_{g}^{\mathrm{eq}}=l_{0}-g /\left(2 \pi f_{k}\right)^{2}$. The stationary solution of Eq. (3) is

$$
\begin{aligned}
z_{g}(t) & =\frac{f_{k}^{2} A}{\sqrt{\left(f_{k}^{2}-f^{2}\right)^{2}+4 a^{2} f^{2(\alpha+1)}}} \cos (2 \pi f t-\phi)+z_{g}^{\mathrm{eq}}, \\
\phi & =\arctan \left(\frac{2 a f^{\alpha+1}}{f_{k}^{2}-f^{2}}\right) .
\end{aligned}
$$

Deriving $z_{g}(t)$, taking the square, and then integrating over a period $1 / f$ we can find the mean quadratic velocity of the particle:

$$
\left\langle\dot{z}_{g}^{2}\right\rangle=\frac{\frac{1}{2} A^{2} f_{k}^{4}(2 \pi f)^{2}}{4 a^{2} f^{2(\alpha+1)}+\left(f_{k}^{2}-f^{2}\right)^{2}} .
$$

As shown in Fig. 10 for the specific value $\alpha=2 / 3 \sim 0.67$ taken from the behavior of $\tau_{c}$ (but it holds in general for $\alpha>0),\left\langle\dot{z}_{g}{ }^{2}\right\rangle$ shows a nonmonotonic behavior for $f<f_{k}$ and its maximum value shifts to the left as $a$ is increased. In Fig. 10 we show the behavior of the mean total kinetic energy $K=m_{\text {eff }}\left\langle\dot{z}_{g}{ }^{2}\right\rangle / 2$ as a function of $S$, for several values of $a$. From a fitting procedure, we found $2 \pi f_{k}=11905 \mathrm{~s}^{-1}$, which is of the order $\sqrt{k / m}$ if the effective stiffness $k$ is estimated by considering series-parallel microscopic elastic constants relative to the grain material, as illustrated above. The prefactor $m_{\text {eff }}$ represents an effective mass and we find an optimal agreement with data for $m_{\mathrm{eff}}=912.6 \mathrm{~g}$, which is of the order of the total mass of the system. Therefore, the analytical model predicts the general phenomenology of the

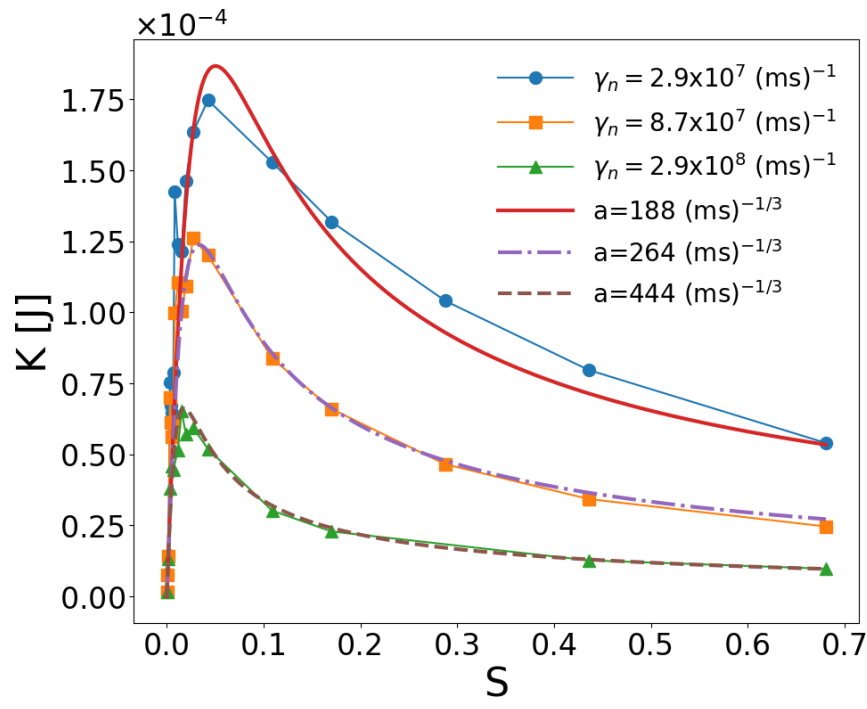

FIG. 10. Mean kinetic energy from Eq. (5) for different values of $a$, with $2 \pi f_{k}=11905 \mathrm{~s}^{-1}, A=0.026 \mathrm{~mm}$, and $m_{\mathrm{eff}}=912.6 \mathrm{~g}$, compared with $K$ measured in numerical simulations with $A=0.026 \mathrm{~mm}$ and $k_{n}=6.1 \times 10^{7} \mathrm{~Pa}$.

three-dimensional granular system, with a good quantitative agreement for high values of $\gamma_{n}$.

\section{B. Beyond a simple resonance}

Equation (5) has a very simple form and its nonmonotonic behavior can be explained in terms of competitive mechanisms. From Eq. (5) we see that the mean quadratic velocity of the particle is proportional to the input energy of the shaker divided by an adimensional factor: $\left\langle\dot{z}_{g}{ }^{2}\right\rangle=V(f) / U(f, \alpha)$, where $V(f)=\frac{1}{2} A^{2}(2 \pi f)^{2}$ is proportional to the strength of the shaker $S=(2 \pi f)^{2} A^{2} /(g d)$, while $U(f, \alpha)=u_{1}+u_{2}$. Here $u_{1}(f, \alpha)=4 a^{2} f_{k}^{-4} f^{2(\alpha+1)}$ and $u_{2}(f)=\left(1-f^{2} / f_{k}^{2}\right)^{2}$ can be considered the two competitive terms if $f<f_{k}$. In this regime, $u_{2}$ is a decreasing function of $f$ and enhances energy transfer, while $u_{1}$ (which contains the dissipation) increases with $f$ and therefore has an opposite effect. In order to better understand the underlying mechanisms, we recall that for an ordinary driven-damped oscillator (namely, with a viscous coefficient that does not depend on $f$ ) the mean quadratic velocity is the same as Eq. (5), with the only difference that the dissipative term is proportional to the square of the rescaled driving frequency: $u_{1}=u_{1}(f, 0)$. From this point of view, $1 / u_{2}$ can be interpreted as an energy gain factor: It increases before the resonant frequency $\left(f=f_{k}\right)$ and then decreases. In the case of the usual damped oscillator, therefore, the nonmonotonic behavior is entirely explained by the nonmonotonic behavior of $u_{2}$ alone, and in fact dissipation does not change the peak position but only smoothes it. On the contrary, when $\alpha>0$ the nonmonotonic behavior has a different origin, coming from the competition between dissipation $\left(u_{1}\right)$ and gain $\left(u_{2}\right)$. This can be rigorously checked, deriving Eq. (5) to find the condition for the maximum,

$$
f_{k}^{4}-f^{4}-4 \alpha a^{2} f^{2(\alpha+1)}=0,
$$


which turns out to be satisfied by $f=f_{k}$ only for $\alpha=0$. The competition is apparently present also in the ordinary driven-damped oscillator but in that case it is balanced by the $(2 \pi f)^{2}$ contained in $V(f)$ in the numerator in such a way that the nonmonotonic behavior of the energy can be explained only by the resonance. We finally note that another way to see competitive terms in Eq. (5) is to rewrite it as $\left\langle\dot{z}_{g}{ }^{2}\right\rangle=\left(u_{1} / V+u_{2} / V\right)^{-1}$. In this form we have the inverse of a sum of two terms that, for $f<f_{k}$, depend in opposite ways and with different powers on $f$. This clearly gives rise to an extremal point also in the limit $f \ll f_{k}$, which is consistent with the values of the fitted parameters.

We stress that these mechanisms substantially differ from the standard resonance phenomenon, due to the presence of the damping term $f_{s} \sim a f^{\alpha}$. Indeed, an increase in $f$ induces the grains to adsorb the injected energy in a faster vibrating motion, experiencing a larger number of collisions (dissipative events) per unit of time. This means that at higher $f$, both the energy input and the energy output increase. The two phenomena compete, and because of their different functional dependencies upon $f$, an extremal point appears. We note that the maxima of the curves in Figs. 2 and 10 occur at frequencies much lower than the fitted $f_{k}$. Conversely, the ordinary resonance always occurs at the fixed characteristic frequency $f_{k}$, independently of the viscous coefficient. For $\alpha>0$, the dissipation controls the position of the peak, reproducing the key feature of molecular dynamic simulations.

\section{COMPARISON WITH PREVIOUS STUDIES}

It is interesting to discuss some previous results [30,31] on energy transfer optimization in vibrated granular media to compare with those presented in this paper. In [30] the authors present experimental and numerical data for a vertically shaken granular system in a three-dimensional geometry. They find a behavior of the kinetic energy $K$ versus the driving frequency $f$ similar to that found by us. Their study is done for increasing $f$ and decreasing $A$, keeping constant the shaker strength $S=(2 \pi f A)^{2} /(g d)$. The main result is that there is a specific combination of $f$ and $A$ that, at a given fixed $S$, optimizes the energy transfer between the shaker and the granular bed. This nonmonotonic trend is rationalized by noting the following competitive effects: on the one hand, raising $f$ increases the maximum rescaled acceleration $\Gamma=(2 \pi f)^{2} A / g$, enhancing the fluidization of the granular medium; on the other hand, decreasing $A$ lowers the mean number of collisions with the shaker, weakening the interaction with the external source of energy.

Regarding [31], the authors study the same setup but concentrating particularly on a one-dimensional geometry, i.e., vertically shaken columns of single grains. In this situation, when $K$ is plotted against $f$ at fixed $S$ they find many peaks at the integer multiples of a specific frequency $f^{*}$. They interpret this phenomenon as a resonant behavior: when the driving frequency is synchronized with the typical time of detachment $\left(\tau^{*}=1 / f^{*}\right)$ of the granular column, the energy transfer from the shaker to the system is optimized.

In the following, we comment on some crucial evidence about the differences between the results reported in our study and those in Refs. [30,31].

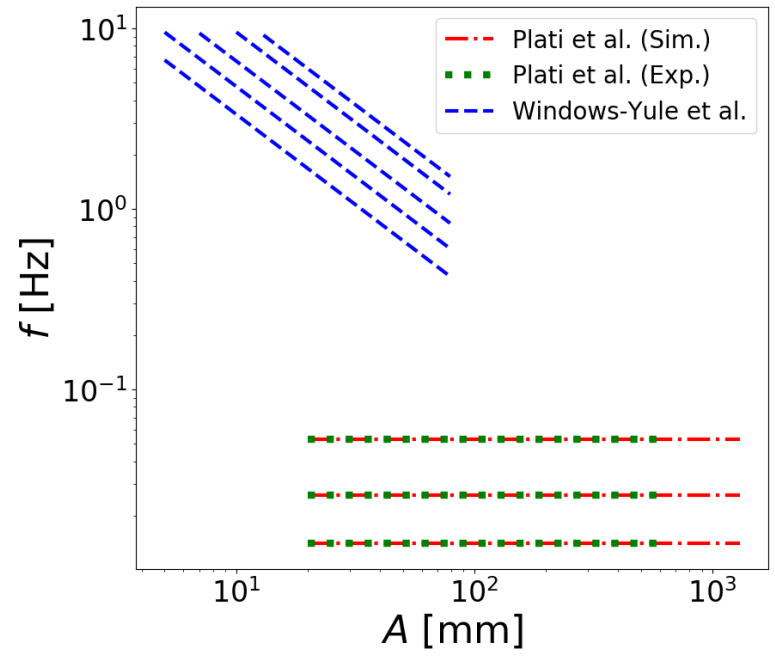

FIG. 11. Different regions of the parameter space investigated in our work with respect to Refs. [30,31].

\section{A. A different granular phase}

Previous studies consider a range of parameters which is completely different from the one considered by us. More precisely, in our experiments/simulations we consider $f \in$ $[20,1300] \mathrm{Hz}$ and $A=0.014,0.026,0.039$, and $0.053 \mathrm{~mm}$, so that $S \in[0.0003,1.15]$; on the contrary, Refs. [30,31] investigate the ranges $f \in[5,80] \mathrm{Hz}$ and $A \in[0.6,8.0] \mathrm{mm}$, corresponding to $S \in[0.9,11.5]$ (see Fig. 11). They consider amplitudes $A$ much higher than those studied in our system. For those values of $A$ the system detaches from the driving plate, reaching the usually called bouncing-bed state, where it is possible to define the time of free flight $\tau^{*}$ responsible for the aforementioned resonant behavior. We also stress that in [30] the granular medium is in a dilute phase where every grain explores all the accessible space, whereas in [31] the grains are arranged in a dense state and each particle in the column remains in the same position with respect to the others.

In our article we show that a vibrofluidized state exists at much smaller values of $A$ (see Fig. 11), sufficiently small to keep the granular medium always in contact with the bottom of the container. This is supported by the collision rate as a function of $f$, plotted in Fig. 12. In our system, indeed, the collision rate is an almost linearly increasing function of $f$, in sharp contrast with the explanation of the phenomenon reported in Refs. [30,31]. More precisely we have checked (visually in the experiments and quantitatively in the simulations) that, for all the driving parameters we explored, the grains are always arranged in a dense packing where they vibrate around an almost-fixed position experiencing rare and slow rearrangements. As a consequence, in the considered range of parameters, the notion of time of free flight is meaningless and the mechanism responsible for the nonmonotonic behavior of $K$ is not related to a resonant mechanism. Indeed, at variance with the bouncing-bed state, the permanent contact allows the oscillating driving plate to continuously transfer kinetic energy to the granular medium as the driving frequency is increased. In our case, the nonmonotonicity of $K$ originates from a competition between the driving energy and 


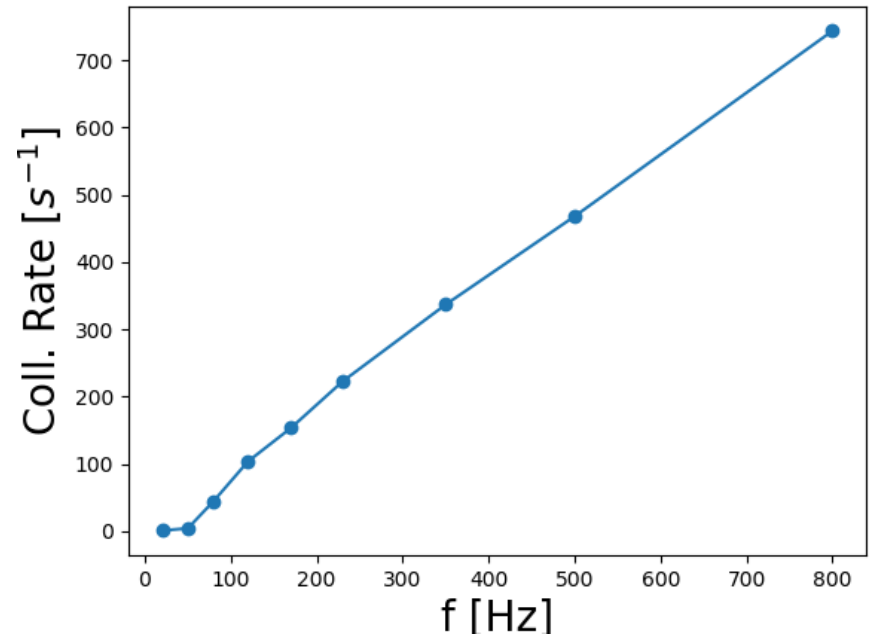

FIG. 12. Collision rate between grains and bottom plate as a function of $f$ with $A=0.026 \mathrm{~mm}$.

the dissipation, which both increase with the frequency, but with different powers of $f$. In this sense, taking the point of view of the authors of [30,31], we observe a maximum of the internal granular energy even if the input energy is increasing.

\section{B. A different role of dissipation}

In the bouncing-bed state the optimal frequency $f^{*}$ is obtained when the vibration period $\tau^{*}$ is synchronized with the flight time of the granular bed, leading to Eq. (5) in Ref. [31],

$$
\tau^{*}=\frac{2 \pi f A}{g}\left\{2+\beta \epsilon \exp \left[-\left(1-\epsilon^{2}\right)(N-1)\right]\right\},
$$

where $g$ is the gravitational acceleration, $\epsilon$ the restitution coefficient (i.e., the fraction of energy lost during a collision), $N$ the number of grains, and $\beta$ a fitting parameter that depends on the details of the experimental setup. Equation (7) clearly shows that the resonant frequency $f^{*}=1 / \tau^{*}$ increases when the restitution coefficient is decreased: the authors rationalize this observation with the fact that more dissipated energy in collisions implies shorter flights, i.e., smaller $\tau^{*}$ or higher resonant frequencies $f^{*}$. In our case, conversely, our peak frequency decreases when the friction coefficient $\gamma_{n}$ of the normal force between the grains (and between grains and borders) is increased. This is the evidence of a very different mechanism at work: when we increase $\gamma_{n}$, i.e., the dissipation per single collision, the range of frequencies where dissipation wins the competition is larger, and this corresponds to a shift of the peak to the left.

\section{Spectra of the top plate}

In Fig. 13, we show the spectra of the $z$ coordinate (measured with a laser sensor; see [33] for details) of the top plate for different values of the driving frequency $f$ and $A=$ $0.053 \mathrm{~mm}$. This figure sheds light on the relation between chaotic motion and energy transfer in our system. We see that the most coherent motion (pronounced peaks in the driving frequency and its harmonics) is obtained for $f=53 \mathrm{~Hz}$ [Fig. 13(a)], before the energy maximum. At the driving
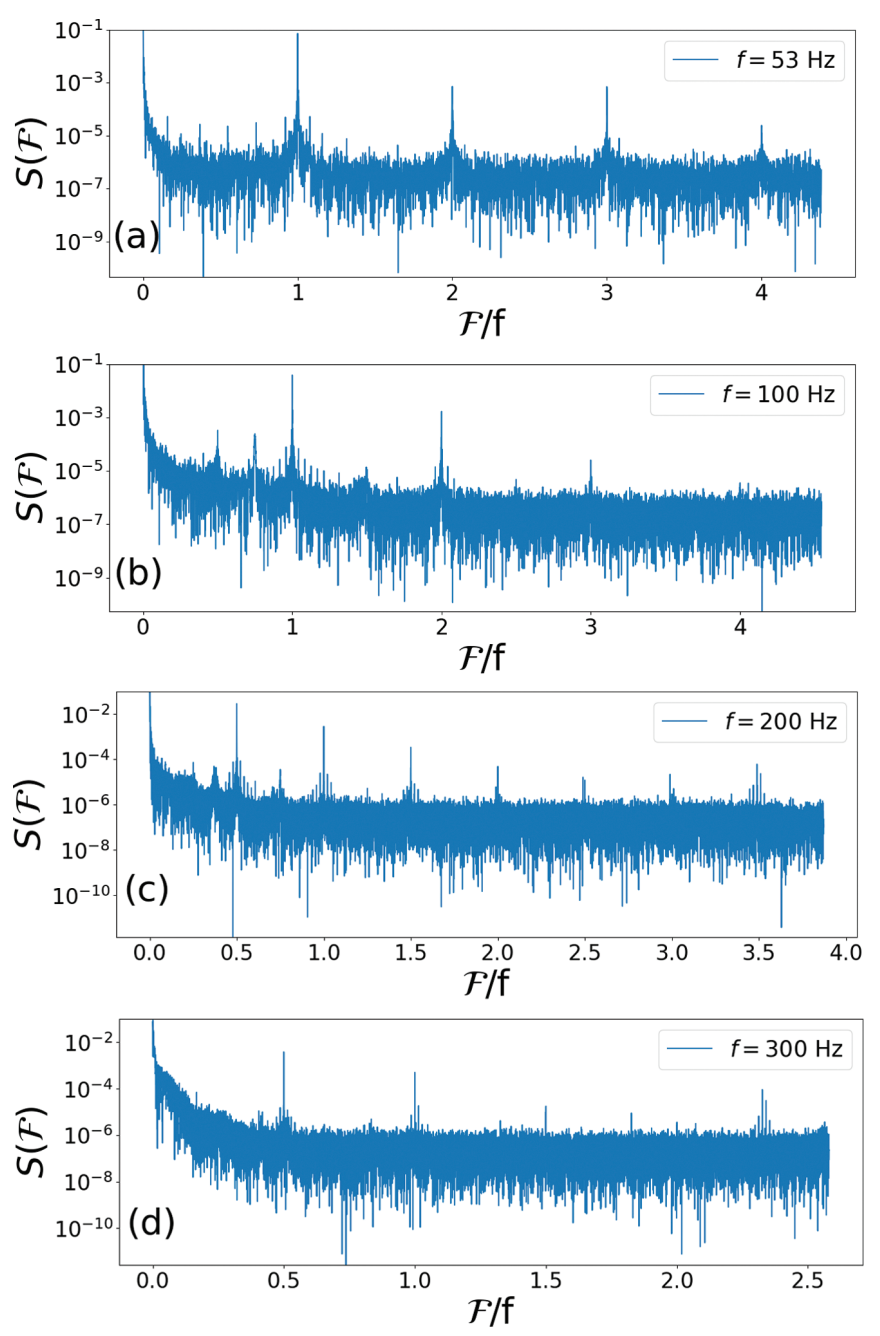

FIG. 13. Experimental data for the spectra of the vertical $z$ coordinate of the top plate for different shaking frequencies $f$ and $A=0.053 \mathrm{~mm}$.

frequency where our energy maximum occurs [Fig. 13(b); $f=100 \mathrm{~Hz}$, we see that the peaks of the harmonics are less pronounced and more broadband. For increasing driving $f$ (decreasing transferred energies), we see a more and more chaotic spectrum, with the appearance of distinct peaks at noninteger multiples of $f$ [Fig. 13(c)] and the disappearance of the peaks in the harmonics [Fig. 13(d)]. Our scenario, therefore, is that of an increasing chaoticity-with $f$-of the granular dynamics, irrespective of the energy transfer, i.e., both before and after the energy maximum. This is quite different from the one observed in [30,31], where the energy transfer to the system is optimized at the frequency such that the granular system is more regular and shows the most defined peaks in its spectrum.

\section{CONCLUSION}

Counterintuitive phenomena, forbidden by standard thermodynamic arguments, can occur in nonequilibrium systems. Negative differential mobility and negative specific heat are typical examples [16,17,22,24]. This kind of nonmonotonic behavior in the current-force relation is due to the 
TABLE I. Numerical values for the material properties and the coefficients of the viscoelastic interaction. We use superscript $a$ for grain-grain, grain-vane, and grain-lid interactions, while superscript $b$ is used for grain-wall ones.

\begin{tabular}{lc}
\hline \hline$k_{n}^{a}$ & $(0.3-1.2) \times 10^{8} \mathrm{~Pa}$ \\
$k_{n}^{b}$ & $0.6 \times 10^{8} \mathrm{~Pa}$ \\
$k_{t}^{a}=k_{t}^{b}$ & $1.3 \times 10^{8} \mathrm{~Pa}$ \\
$\gamma_{n}^{a}=\gamma_{n}^{b}$ & $2.9 \times\left(10^{5}-10^{8}\right)(\mathrm{ms})^{-1}$ \\
$\gamma_{t}^{a}=\gamma_{t}^{b}$ & $2.3 \times\left(10^{3}-10^{6}\right)(\mathrm{ms})^{-1}$ \\
$\mu^{a}=\mu^{b}$ & 0.5 \\
$d t$ & $2.7 \times 10^{-6} \mathrm{~s}$ \\
\hline \hline
\end{tabular}

combination of competing mechanisms. Here we have unveiled a similar effect in a complex many-body system with dissipative interactions: The interplay between external forcing and internal dissipation in granular media can result in a nonmonotonic behavior of the system kinetic energy as a function of the input energy, representing an instance of negative specific heat. Moreover, our analysis explains the important role played by the vibration frequency, triggering specific dissipation mechanisms. These arise at different scales, from rheological behavior to single-particle motion. The observed phenomenology may have a deep impact application in several fields related to granular matter physics.

\section{ACKNOWLEDGMENTS}

E.L., A.P., and A.S. acknowledge support from MIUR PRIN 2017 project 201798CZLJ. L.d.A. acknowledges support from project PRIN2017WZFTZP. L.d.A., E.L., and A.S. acknowledge support from the VALERE project of the University of Campania “L. Vanvitelli.” A. Plati, A.P., and A.G. acknowledge support from Regione Lazio through the Grant "Progetti Gruppi di Ricerca" (No. 85-2017-15257).

\section{APPENDIX: SIMULATION DETAILS}

Our molecular dynamics simulations are performed using the LAMMPS package [48] and the system geometry reproduces the experimental setup in Ref. [33]. The values of $\langle\Omega\rangle$ and $K$ reported in the text are the result of a time average over $3 \times 10^{7}$ time steps (78 equivalent s) at steady state. Regarding the interaction between the grains, we used the Hertz-Mindlin model $[49,50,56]$ to solve the dynamics during the contact. This viscoelastic model takes into account both the elastic and the dissipative response to the mutual compression. In addition, it includes in the dynamics not only the relative translational motion but also the rotational one. The model is described by the following equations:

$$
\begin{aligned}
\vec{F}_{i j}^{N} & =\sqrt{R_{i j}^{\mathrm{eff}}} \sqrt{\xi_{i j}(t)}\left[\left(k_{n} \xi_{i j}(t)-m^{\mathrm{eff}} \gamma_{n} \dot{\xi}_{i j}(t)\right) \vec{n}(t)\right] ; \\
\vec{F}_{i j}^{T} & =\left\{\begin{array}{l}
-\sqrt{R_{i j}^{\mathrm{eff}}}\left[\vec{F}_{i j}^{\text {hist }}+m_{\mathrm{eff}} \gamma_{t} \sqrt{\xi_{i j}(t)} \vec{g}_{i j}^{T}(t)\right] \text { if }\left|\vec{F}_{i j}^{\mathrm{hist}}\right| \leqslant\left|\mu \vec{F}_{i j}^{N}\right|, \\
-\frac{\left|\mu \vec{F}_{i j}^{N}\right|}{\left|\vec{g}_{i j}^{T}(t)\right|} \cdot \vec{g}_{i j}^{T}(t) \quad \text { otherwise; }
\end{array}\right. \\
\vec{F}_{i j}^{\text {hist }} & =k_{t} \int_{\mathrm{s}(\mathrm{t})} \sqrt{\xi_{i j}\left(t^{\prime}\right)} \vec{d} s\left(t^{\prime}\right) .
\end{aligned}
$$

These equations are written for two particles with radii $R_{i}$ and $R_{j}$, center positions $\vec{r}_{i}$ and $\vec{r}_{j}$, translational velocities $\vec{v}_{i}$ and $\vec{v}_{j}$, and rotational velocities $\vec{\omega}_{i}$ and $\vec{\omega}_{j}$. The relative velocity is defined as $\vec{g}_{i j}=\left(\dot{\vec{r}}_{i}-\vec{\omega}_{i} \times R_{i} \vec{n}\right)-\left(\dot{\vec{r}}_{j}+\vec{\omega}_{j} \times R_{j} \vec{n}\right)$, where $\vec{n}=\left(\vec{r}_{i}-\vec{r}_{j}\right) /\left|\vec{r}_{i}-\vec{r}_{j}\right|$; we call $\vec{g}_{i j}^{N}$ and $\vec{g}_{i j}^{T}$ the two projections normal and tangential, respectively, to the surface of contact. The instantaneous normal compression is represented by $\xi_{i j}(t)=R_{i}+R_{j}-\left|\vec{r}_{i}-\vec{r}_{j}\right|$ and its derivative is $\dot{\xi}_{i j}(t)=\left|\vec{g}_{i j}^{N}\right|$. During the contact, the particles are subjected to a normal force $\vec{F}_{i j}^{N}$ and a tangential one $\vec{F}_{i j}^{T}$; both these components have an elastic and a dissipative contribution characterized, respectively, by the coefficients $k_{n}, k_{t}, \gamma_{n}$, and $\gamma_{t}$. In the normal force $\vec{F}_{i j}^{N}$ we can see an elastic term that derives from the hertzian theory of contact mechanics [57] characterized by a nonlinear dependence on the displacement. The HM model is used to describe the interactions between all elements of the simulation (the box, the vane, and the lid), considering the flat surfaces of the box as spheres with infinite mass and radius. Further discussions of the physical meaning and estimation of the model parameters and of the choice of the simulation time step $d t$ can be found in the Supplemental Material to [54]. Here we report the numerical values used in the present study (Table I).
[1] H. M. Jaeger, S. R. Nagel, and R. P. Behringer, Granular solids, liquids, and gases, Rev. Mod. Phys. 68, 1259 (1996).

[2] A. P. J. Breu, H.-M. Ensner, C. A. Kruelle, and I. Rehberg, Reversing the Brazil-Nut Effect: Competition Between Percolation and Condensation, Phys. Rev. Lett. 90, 014302 (2003).

[3] D. van der Meer, P. Reimann, K. van der Weele, and D. Lohse, Spontaneous Ratchet Effect in a Granular Gas, Phys. Rev. Lett. 92, 184301 (2004).

[4] A. Gnoli, A. Petri, F. Dalton, G. Pontuale, G. Gradenigo, A. Sarracino, and A. Puglisi, Brownian Ratchet in a Thermal Bath Driven by Coulomb Friction, Phys. Rev. Lett. 110, 120601 (2013).
[5] M. Schröter, S. Ulrich, J. Kreft, J. B. Swift, and H. L. Swinney, Mechanisms in the size segregation of a binary granular mixture, Phys. Rev. E 74, 011307 (2006).

[6] V. Garzó and E. Trizac, Non-equilibrium phase transition in a sheared granular mixture, Europhys. Lett. 94, 50009 (2011).

[7] J. A. C. Gallas, H. J. Herrmann, and S. Sokołowski, Convection Cells in Vibrating Granular Media, Phys. Rev. Lett. 69, 1371 (1992).

[8] G. Pontuale, A. Gnoli, F. Vega Reyes, and A. Puglisi, Thermal Convection in Granular Gases with Dissipative Lateral Walls, Phys. Rev. Lett. 117, 098006 (2016). 
[9] A. Prados and E. Trizac, Kovacs-Like Memory Effect in Driven Granular Gases, Phys. Rev. Lett. 112, 198001 (2014).

[10] I. S. Aranson and L. S. Tsimring, Patterns and collective behavior in granular media: Theoretical concepts, Rev. Mod. Phys. 78, 641 (2006).

[11] A. Puglisi, A. Sarracino, and A. Vulpiani, Temperature in and out of equilibrium: A review of concepts, tools and attempts, Phys. Rep. 709, 1 (2017).

[12] R. L. Jack, D. Kelsey, J. P. Garrahan, and D. Chandler, Negative differential mobility of weakly driven particles in models of glass formers, Phys. Rev. E 78, 011506 (2008).

[13] M. Sellitto, Asymmetric Exclusion Processes with Constrained Dynamics, Phys. Rev. Lett. 101, 048301 (2008).

[14] S. Leitmann and T. Franosch, Nonlinear Response in the Driven Lattice Lorentz Gas, Phys. Rev. Lett. 111, 190603 (2013).

[15] P. Baerts, U. Basu, C. Maes, and S. Safaverdi, Frenetic origin of negative differential response, Phys. Rev. E 88, 052109 (2013).

[16] O. Bénichou, P. Illien, G. Oshanin, A. Sarracino, and R. Voituriez, Microscopic Theory for Negative Differential Mobility in Crowded Environments, Phys. Rev. Lett. 113, 268002 (2014).

[17] A. Sarracino, F. Cecconi, A. Puglisi, and A. Vulpiani, Nonlinear Response of Inertial Tracers in Steady Laminar Flows: Differential and Absolute Negative Mobility, Phys. Rev. Lett. 117, 174501 (2016).

[18] D. Lynden-Bell, Negative specific heat in astronomy, physics and chemistry, Phys. A 263, 293 (1999).

[19] J. Barré, D. Mukamel, and S. Ruffo, Inequivalence of Ensembles in a System with Long-Range Interactions, Phys. Rev. Lett. 87, 030601 (2001).

[20] A. Ramírez-Hernández, H. Larralde, and F. Leyvraz, Violation of the Zeroth Law of theRmodynamics in Systems with Negative Specific Heat, Phys. Rev. Lett. 100, 120601 (2008).

[21] F. Staniscia, A. Turchi, D. Fanelli, P. H. Chavanis, and G. De Ninno, Negative Specific Heat in the Canonical Statistical Ensemble, Phys. Rev. Lett. 105, 010601 (2010).

[22] R. K. P. Zia, E. L. Praestgaard, and O. G. Mouritsen, Getting more from pushing less: Negative specific heat and conductivity in nonequilibrium steady states, Am. J. Phys. 70, 384 (2002).

[23] J. Bisquert, Master equation approach to the non-equilibrium negative specific heat at the glass transition, Am. J. Phys. 73, 735 (2005).

[24] N. V. Brilliantov, A. Formella and T. Pöschel, Increasing temperature of cooling granular gases, Nat. Commun. 9, 797 (2018).

[25] P. Coussot, Rheometry of Pastes, Suspensions, and Granular Materials: Applications in Industry and Environment (John Wiley \& Sons, New York, 2005).

[26] S. Luding, E. Clement, A. Blumen, J. Rajchenbach, and J. Duran, Studies of columns of beads under external vibrations, Phys. Rev. E 50, 4113 (1994).

[27] S. McNamara and S. Luding, Energy flows in vibrated granular media, Phys. Rev. E 58, 813 (1998).

[28] V. Kumaran, Temperature of a granular material fluidized by external vibrations, Phys. Rev. E 57, 5660 (1998).

[29] V. Zivkovic, M. J. Biggs, and D. H. Glass, Scaling of granular temperature in a vibrated granular bed, Phys. Rev. E 83, 031308 (2011).
[30] C. R. K. Windows-Yule, A. D. Rosato, D. J. Parker, and A. R. Thornton, Maximizing energy transfer in vibrofluidized granular systems, Phys. Rev. E 91, 052203 (2015).

[31] C. R. K. Windows-Yule, A. D. Rosato, D. J. Parker, and A. R. Thornton, Resonance effects on the dynamics of dense granular beds: achieving optimal energy transfer in vibrated granular systems, New J. Phys. 17, 023015 (2015).

[32] H. Lastakowski, J.-C. Géminard, and V. Vidal, Granular friction: Triggering large events with small vibrations, Sci. Rep. 5, 13455 (2015).

[33] A. Gnoli, L. de Arcangelis, F. Giacco, E. Lippiello, M. P. Ciamarra, A. Puglisi, and A. Sarracino, Controlled Viscosity in Dense Granular Materials, Phys. Rev. Lett. 120, 138001 (2018).

[34] V. Vidal, C. Oliver, H. Lastakowski, G. Varas, and J.-C. Géminard, Friction weakening by mechanical vibrations: A velocity-controlled process, Eur. Phys. J. E 42, 91 (2019).

[35] J. A. Aström, H. J. Herrmann, and J. Timonen, Granular Packings and Fault Zones, Phys. Rev. Lett. 84, 638 (2000).

[36] M. Urbakh, J. Klafter, D. Gourdon, and J. Israelachvili, The nonlinear nature of friction, Nature 430, 525 (2004).

[37] K. E. Daniels and R. P. Behringer, Hysteresis and Competition Between Disorder and Crystallization in Sheared and Vibrated Granular Flow, Phys. Rev. Lett. 94, 168001 (2005).

[38] P. A. Johnson and X. Jia, Nonlinear dynamics, granular media and dynamic earthquake triggering, Nature 437, 871 (2005).

[39] R. Capozza, A. Vanossi, A. Vezzani, and S. Zapperi, Suppression of Friction by Mechanical Vibrations, Phys. Rev. Lett. 103, 085502 (2009).

[40] J. A. Dijksman, G. H. Wortel, L. T. van Dellen, O. Dauchot, and M. van Hecke, Jamming, Yielding, and Rheology of Weakly Vibrated Granular Media, Phys. Rev. Lett. 107, 108303 (2011).

[41] A. L. Sellerio, D. Mari, G. Gremaud, and G. D'Anna, Glass transition associated with the jamming of vibrated granular matter, Phys. Rev. E 83, 021301 (2011).

[42] M. Griffa, B. Ferdowsi, R. A. Guyer, E. G. Daub, P. A. Johnson, C. Marone, and J. Carmeliet, Influence of vibration amplitude on dynamic triggering of slip in sheared granular layers, Phys. Rev. E 87, 012205 (2013).

[43] B. A. Klumov, Y. Jin, and H. A. Makse, Structural properties of dense hard sphere packings, J. Phys. Chem. B 118, 10761 (2014).

[44] G. H. Wortel, J. A. Dijksman, and M. van Hecke, Rheology of weakly vibrated granular media, Phys. Rev. E 89, 012202 (2014).

[45] F. Giacco, L. Saggese, L. de Arcangelis, E. Lippiello, and M. Pica Ciamarra, Dynamic Weakening by Acoustic Fluidization During Stick-Slip Motion, Phys. Rev. Lett. 115, 128001 (2015).

[46] P. Charbonneau, E. I. Corwin, G. Parisi, and F. Zamponi, Jamming Criticality Revealed by Removing Localized Buckling Excitations, Phys. Rev. Lett. 114, 125504 (2015).

[47] A. Gnoli, A. Lasanta, A. Sarracino, and A. Puglisi, Unified rheology of vibro-fluidized dry granular media: From slow dense flows to fast gas-like regimes, Sci. Rep. 6, 38604 (2016). 
[48] S. Plimpton, Fast parallel algorithms for short-range molecular dynamics, J. Comput. Phys. 117, 1 (1995).

[49] L. E. Silbert, D. Ertaş, G. S. Grest, T. C. Halsey, D. Levine, and S. J. Plimpton, Granular flow down an inclined plane: Bagnold scaling and rheology, Phys. Rev. E 64, 051302 (2001).

[50] N. V. Brilliantov, F. Spahn, J.-M. Hertzsch, and T. Pöschel, Model for collisions in granular gases, Phys. Rev. E 53, 5382 (1996).

[51] Since we are mainly interested in the study of the total granular kinetic energy, we focus on the vane velocity fluctuations around the average, subtracting the contribution due to the external forcing imposed by the motor. As shown in the rest of the paper, $K_{v}$ is a good proxy for the global granular energy.

[52] We have verified that the presence of the vane does not significantly affect the granular features.
[53] C. Scalliet, A. Gnoli, A. Puglisi, and A. Vulpiani, Cages and Anomalous Diffusion in Vibrated Dense Granular Media, Phys. Rev. Lett. 114, 198001 (2015).

[54] A. Plati, A. Baldassarri, A. Gnoli, G. Gradenigo, and A. Puglisi, Dynamical Collective Memory in Fluidized Granular Materials, Phys. Rev. Lett. 123, 038002 (2019).

[55] F. Giacco, L. de Arcangelis, M. Pica Ciamarra, and E. Lippiello, Rattler-induced aging dynamics in jammed granular systems, Soft Matter 13, 9132 (2017).

[56] H. P. Zhang and H. A. Makse, Jamming transition in emulsions and granular materials, Phys. Rev. E 72, 011301 (2005).

[57] V. L. Popov, Contact Mechanics and Friction (Springer-Verlag, Berlin, 2010)

Correction: The plots for panels (a) and (b) in the previously published Figure 3 were erroneously inverted and have been fixed. 